ARTICLE

Received 12 Apr 2014 | Accepted 30 Jul 2014 | Published 11 Sep $2014 \quad$ DOl: 10.1038/ncomms5850

\title{
Ribosomal DNA copy number is coupled with gene expression variation and mitochondrial abundance in humans
}

\author{
John G. Gibbons ${ }^{1}$, Alan T. Branco', Shoukai Yu \& Bernardo Lemos ${ }^{1}$
}

Ribosomes are essential intracellular machines composed of proteins and RNA molecules. The DNA sequences (rDNA) encoding ribosomal RNAs (rRNAs) are tandemly repeated and give origin to the nucleolus. Here we develop a computational method for estimating rDNA dosage (copy number) and mitochondrial DNA abundance using whole-genome short-read DNA sequencing. We estimate these attributes across hundreds of human genomes and their association with global gene expression. The analyses uncover abundant variation in rDNA dosage that is coupled with the expression of hundreds of functionally coherent gene sets. These include associations with genes coding for chromatin components that target the nucleolus, including CTCF and HP1 3 . Finally, the data show an inverse association between rDNA dosage and mitochondrial DNA abundance that is manifested across genotypes. Our findings uncover a novel and cryptic source of hypervariable genomic diversity with global regulatory consequences (ribosomal eQTL) in humans. The variation provides a mechanism for cellular homeostasis and for rapid and reversible adaptation.

\footnotetext{
${ }_{1}^{1}$ Program in Molecular and Integrative Physiological Sciences, Department of Environmental Health, Harvard School of Public Health, 665 Huntington Avenue, Building 2, Room 219, Boston, Massachusetts 02115, USA. Correspondence and requests for materials should be addressed to B.L. (email: blemos@hsph.harvard.edu).
} 
C opy number variation (CNV) is a significant source of natural genetic diversity. The ribosomal RNAs (rRNAs) are essential structural and catalytic components of ribosomes, are conserved throughout all domains of life and are encoded in repetitive loci (ribosomal DNA (rDNA) arrays) that might be among the most copy number $(\mathrm{CN})$ hypervariable genomic segments. However, the functional consequences of rDNA CN polymorphisms are unknown and often assumed to be negligible. The eukaryotic ribosome is an indispensable cellular machine composed of a core complex of $\sim 80$ proteins and 4 rRNA molecules. The mature $80 \mathrm{~S}$ ribosome consists of the small $40 \mathrm{~S}$ (containing the $18 \mathrm{~S}$ rRNA) and large $60 \mathrm{~S}$ (containing the $28 \mathrm{~S}$, $5.8 \mathrm{~S}$ and $5 \mathrm{~S}$ rRNAs) subunits, each with distinct functions ${ }^{1}$. In humans, the 18S, 5.8S and 28S rRNA molecules are encoded within tandemly repeated $43-\mathrm{kb}$ nucleolar organizing regions residing on the five acrocentric autosomal chromosomes (13, 14, $15,21$ and 22$)^{2}$ (Fig. 1), while the $5 \mathrm{~S}$ rRNA is encoded by a tandemly repeated $\sim 2.2-\mathrm{kb}$ cluster on chromosome 1 (1q42 region $)^{3}$. In addition, $\sim 150$ proteins and $\sim 70$ small nucleolar RNAs interact or are associated with the ribosome during its biogenesis $^{4}$. Remarkably, transcription from the rRNAs constitutes over $80 \%$ of cellular RNAs with thousands of ribosomal subunits synthesized each minute in actively growing eukaryotic cells ${ }^{5,6}$. Considering the pivotal role of the ribosome, it is of little surprise that a number of diseases are traced to defective ribosomal proteins and deficient rRNA transcription ${ }^{7}$.
Mitochondria, the organelles responsible for energy production, are also critical for cellular function, contain their own genome $(\sim 16.5 \mathrm{~Kb})$ independent from the nucleus (Fig. 1), are present at variable levels in cells and interact with nuclear ribosomes through coordinated processes ${ }^{8-10}$. Indeed, functional relationships between the rRNA transcription and the mitochondria are evident ${ }^{10-13}$. Finally, analyses in Drosophila indicated that the expression of genes that localize to the mitochondria is responsive to engineered deletions that decreased rDNA $\mathrm{CN}^{8}$. Hence, these observations raise the expectation that the cross-talk between the rDNA dosage and mitochondrial abundance might be manifested across genotypes in natural populations.

Here we develop a method for assessing rDNA dosage and mitochondria abundance using local variation in coverage depth in short-read whole-genome DNA sequence data (Fig. 1). We apply this methodology to human whole-genome sequencing data $^{14}$ and investigate the extent of variation in rDNA dosage (the number of rDNA copies present in a given genome) and mitochondria DNA abundance (the number of mitochondrial genomes present in a cell). Estimates of rDNA dosage and mitochondria (mitochondrial DNA (mtDNA)) abundance are combined with gene expression (GE) estimates from RNA-seq data to reveal the functional consequences of rDNA dosage variation. Our analysis sheds new light on the diverse functional roles of the rDNA loci and uncovers coordinated variation

a

Whole genom

sequencing
illumina reads

C

Read depth calculations
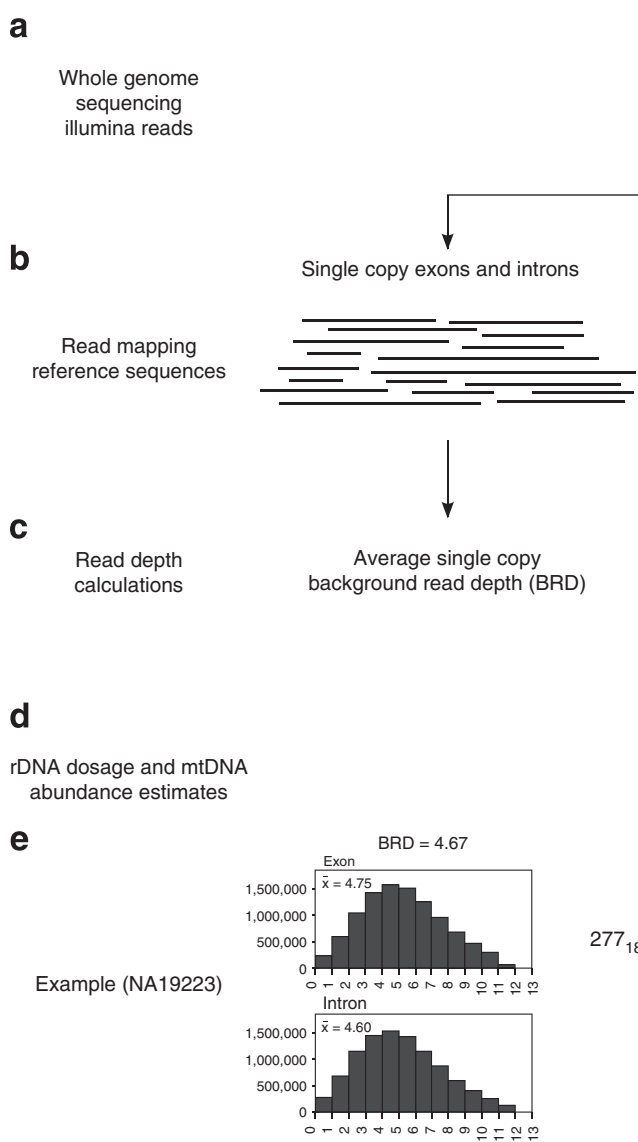

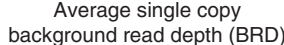

RD)
Genomic reads

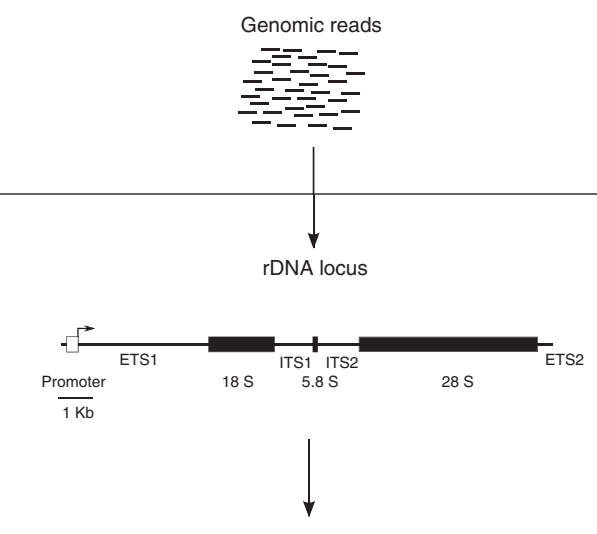

rDNA Component Average Read Depth (ARD)
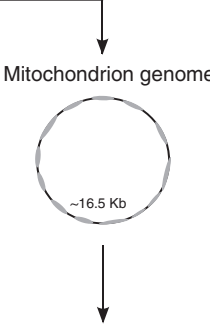

Mitochondrion genome average read depth (ARD)

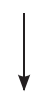

mtDNA abundance $=\frac{\text { mtDNA ARD }}{\text { BRD }}$ rDNA dosage and mtDNA
abundance estimates $\downarrow$

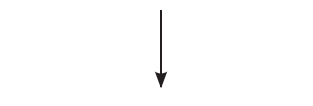

rDNA Dosage $=\frac{\text { rDNA ARD }}{B R D}$

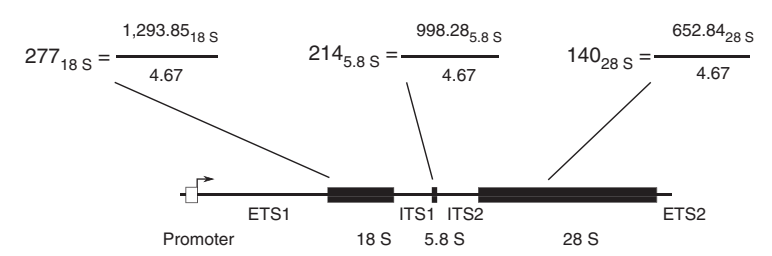

Promoter $18 \mathrm{~S} \quad 5.8 \mathrm{~S}$ $28 \mathrm{~S}$

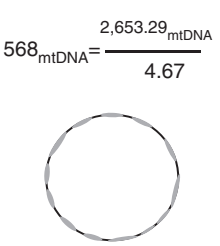

Figure 1 | Experimental procedure to estimate rDNA dosage and mtDNA abundance from short-read whole-genome sequence data. (a, b) Genomic reads were quality trimmed and mapped against a panel of single-copy exons and introns, the rDNA locus and the mitochondrion genome. (c) BRD,

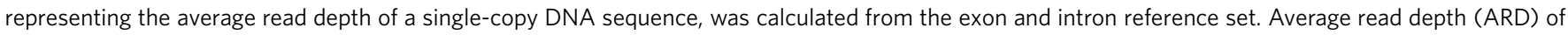
the rDNA locus and mitochondrion genome was calculated. (d) rDNA and mtDNA ARD values were normalized by BRD to estimate dosage and

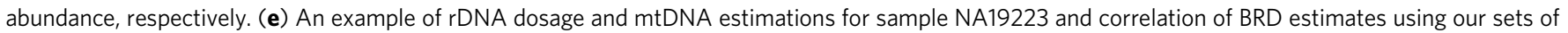
single-copy exon and intron across samples. 
between the rDNA loci and global GE, as well as mitochondrial abundance. The associations are manifested in the context of naturally occurring variation among individuals.

\section{Results}

Estimation of rDNA dosage. Local variation in read depth has been benchmarked as a reliable method for the identification and quantification of $\mathrm{CNV}^{15,16}$. We modified the approach to quantify dosage of the rRNA encoding loci, as the read depth coverage of the rDNA components relative to baseline read depth of single copy sequences (see Fig. 1 for methodological pipeline). Reads for each sample were quality trimmed and mapped against the reference (i) panel of single copy exons $(12,453$ exon totaling $13.09 \mathrm{Mb}$ ), (ii) panel of single copy introns $(1,131$ introns totaling $\sim 1.27 \mathrm{Mb}$ ), (iii) $45 \mathrm{~S}$ rDNA locus (the locus containing the $18 \mathrm{~S}$, $5.8 \mathrm{~S}$ and $28 \mathrm{~S}$ rRNA encoding genes) ${ }^{17}$ and (iv) mitochondrion genome (Fig. 1, see Methods). Per-base read depth values were normally distributed and sample-specific sequence read depth was estimated by the mean base coverage (Fig. 1e). The average per-base single copy exon and single copy intron was used as a proxy for the background read depth (BRD). We focus subsequent analyses on 168 individuals from 2 populations with genomic coverage $>4 \times$ (Supplementary Table 1). Our calculation of $\mathrm{BRD}$ produced nearly identical estimates of genomic coverage for both exon and intron reference data sets $\left(r^{2}=0.99, P=1.5 \times 10^{-173}\right.$; Supplementary Fig. 1$)$. BRD ranged from 4.7 to $16.2 \times$ and, importantly, was not correlated with dosage of any rDNA component. Average per-base read depth of rDNA sequences was normalized by the BRD to estimate rDNA dosage (Fig. 1, see Methods).

Several analyses validate our rDNA dosage estimates. First, we measured $\mathrm{CN}$ for six unrelated genes that were previously characterized $^{16}$ (see Methods). Our results strongly agree with previous estimates across all six genes (average $r^{2}=0.88$; Supplementary Fig. 2), including the two genes with experimental validation (CCL3L1: $r^{2}=0.91, P=2.3 \times 10^{-43}$ and TBC1D3F: $r^{2}=0.81, \quad P=9.8 \times 10^{-30}$; Supplementary Fig. 2a,b). Second, we investigated whether sequence similarity between rDNA components might confound estimates. We observed complete lack of sequence similarity between rDNA components using either BLAST or ClustalW alignments. Conversely, each rDNA component is highly conserved between humans and mouse (Pairwise nucleotide identity: $18 \mathrm{~S}=100 \%, 5.8 \mathrm{~S}=99 \%$ and $28 \mathrm{~S}=86 \%)$. Indeed, inspection of reads mapping to the rDNA revealed that no reads mapped to multiple rDNA components (see Methods; Supplementary Fig. 3). These analyses indicate that the rDNA components did not interfere with one another during read mapping and point to robust computational estimates of rDNA dosage.

Third, estimates of rDNA dosage could be confounded if reads that map to the rDNA loci also map to pseudogenes at alternative regions of the genome. To address the issue, we extracted reads that mapped to each component of the reference rDNA locus and mapped those 'rDNA reads' against the reference GRCh37 (hg19) human genome (see Methods; Fig. 2). The reference genome includes chromosomes and all supercontigs that have not been assigned a chromosomal location. The chromosomal regions containing nucleolar organizing regions are masked in the reference genome. The procedure was independently performed for each of the 168 samples. As expected, the vast majority of $45 \mathrm{~S}$ rDNA reads mapped to a single supercontig that harbours the $45 \mathrm{~S}$ rDNA locus (GL000220.1; 18S: 87.41\%, 5.8S: $98.3 \%$ and $28 \mathrm{~S}$ : $94.4 \%$; Fig. 3a). Hence, for each individual, we used the percentage of reads mapping to the expected chromosomes and contigs as a correction factor to obtain the best estimates of $18 \mathrm{~S}$,
5.8S and 28S dosage. The correction had minor but beneficial effect on individual estimates and resulted in a slightly higher correlation for $18 \mathrm{~S}$ versus $5.8 \mathrm{~S}(r=0.80$ versus $r=0.79)$, and identical correlations for $28 \mathrm{~S}$ versus $18 \mathrm{~S}$ and $28 \mathrm{~S}$ versus $5.8 \mathrm{~S}$. Interestingly, the majority of the $\sim 13 \%$ of $18 \mathrm{~S}$ reads, which did not map to GL000220.1 supercontig, mapped to a small ( $\sim 600 \mathrm{bp})$ region of chromosome $21 \quad(9,826,921-9,827,532$; Fig. $3 \mathrm{~b}$ ). BLAST results indicate that this region, and its flanking regions, share homology with ETS1 and 18S but with no other rDNA elements (Fig. 3b). This suggests that the region is probably a truncated and pseudogenized rDNA array.

rDNA dosage is highly polymorphic. Diploid rDNA dosage was quantified for each coding component of the rDNA locus (18S, $5.8 \mathrm{~S}$ and $28 \mathrm{~S}$ regions). Diploid rDNA dosage for each component ranged from 67 to 412 for $18 \mathrm{~S}(\overline{\mathrm{x}}=217$ copies), 9 to 421 for $5.8 \mathrm{~S}$ $(\overline{\mathrm{x}}=164$ copies $)$ and 26 to 282 for $28 \mathrm{~S}(\overline{\mathrm{x}}=118$ copies $)$. Further, the components of the $45 \mathrm{~S}$ rDNA locus were significantly and strongly correlated with one another (Fig. $2 ; N=168$; $18 \mathrm{~S}$ versus 5.8S: Pearson's $r=0.79, P=2 \times 10^{-37}, 18 \mathrm{~S}$ versus $28 \mathrm{~S}$ : $r=0.85$, $P=2 \times 10^{-47}$ and 5.8 S versus $28 \mathrm{~S}$ : $\left.r=0.96, P=1 \times 10^{-95}\right)$. These patterns were consistent between populations and between sexes (Fig. 2). The associations are expected due to the close physical linkage between the components of the $45 \mathrm{~S}$ rDNA array. Nevertheless, abnormally rearranged rDNA loci might contribute to variability in dosage between rDNA components ${ }^{18-20}$ and might account for the weaker association between the $18 \mathrm{~S}$ and the $35 \mathrm{~S}$ components (5.8S and 28S). In terms of the range of our computational rDNA dosage measurements, our results are consistent with previous experimental estimates of $45 \mathrm{~S}$ dosage measured by the cumulative physical size of the rDNA clusters $^{21,22}$. Collectively, the data point to substantial variation across individuals.

Loci with dosage variation might display variable levels of population differentiation ranging from an absence of population differentiation to high levels of differentiation ${ }^{16}$. To address the issue for the rDNA loci, we estimated $V_{\mathrm{ST}}$ values between Ceph (CEU) and Yoruban (YRI) populations. We observe no evidence for population differentiation within the rDNA loci (18S $V_{\mathrm{ST}}=0.09$, 5.8S $V_{\mathrm{ST}}<0.001$ and $28 \mathrm{~S} \quad V_{\mathrm{ST}}=0.01$; Fig. 4a-c). Principal component analyses (PCAs) of the rDNA dosage components further reinforce the conclusion that these populations are not differentiated in regards to rDNA dosage (Fig. 4d,e).

The rDNA is a trans-regulatory eQTL. Recent data suggested that rDNA deletions might disrupt GE levels and chromatin states in Drosophila ${ }^{8,23}$. Substantial association in dosage among unlinked loci further lends support to the hypothesis that variability at the rDNA loci might have functional relevance for GE states across the genome. Accordingly, we hypothesized that rDNA dosage might function as expression quantitative trait loci (eQTL) modulating natural variation in GE levels among individuals. To address the issue, we cross-referenced our estimates of rDNA dosage with genome-wide GE data from the HapMap project ${ }^{24,25}$ (see Methods). The combined data set consists of 97 individuals with corresponding rDNA dosage estimates and whole-genome expression data from lymphoblastoid cell lines (Supplementary Table 1). Genomewide association between rDNA dosage estimates and GE levels was calculated for each rDNA component independently using Pearson's correlation and, formally, through mixed linear models (Fig. 5). These analyses revealed highly statistically significant and comparable associations across the $18 \mathrm{~S}, 5.8 \mathrm{~S}$ and $28 \mathrm{~S} \mathrm{rDNA}$ components (as expected due to strong rDNA dosage correlations 
a

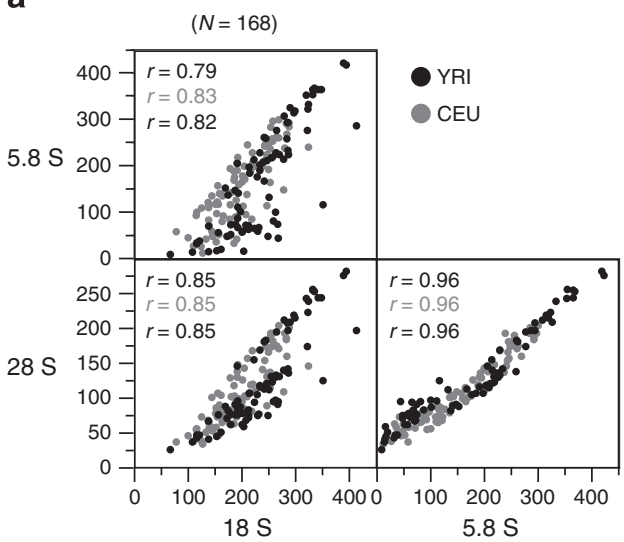

b

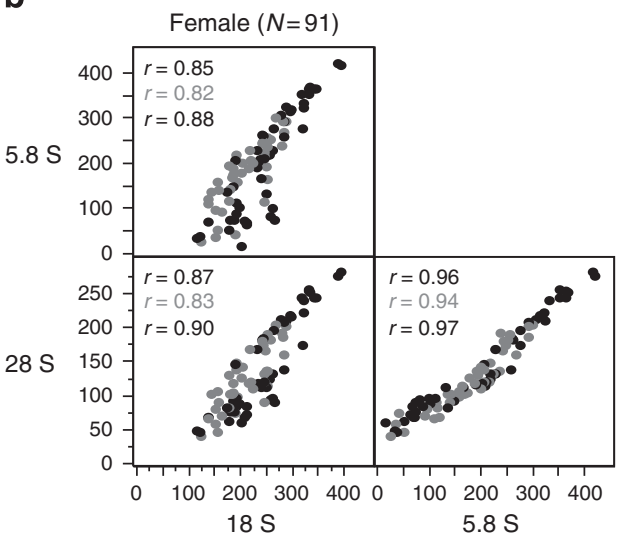

C

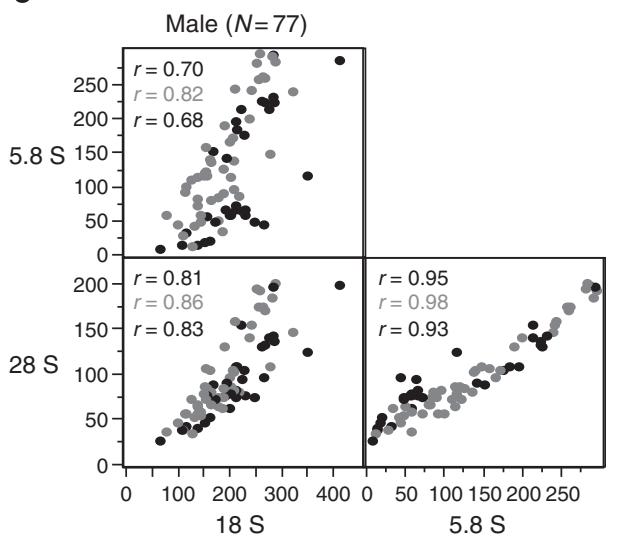

Figure 2 | Dosage estimates for the three components of the 45S rDNA are highly correlated with one another. (a) Pairwise Pearson's correlation ( $r$ ) for the $45 \mathrm{~S}$ rDNA components $(N=168)$. For all correlations $P$-value $\leq 2 \times 10^{-37}$. Grey and black points represent the CEU and YRI populations, respectively. $(\mathbf{b}, \mathbf{c})$ Correlations are consistent when stratified by sex. For all comparisons, the bold Pearson value corresponds to the combined data set, while the grey and black Pearson values represent the CEU and YRI populations, respectively. Dosage is estimated as the number of rDNA copies per diploid genome.

between components; Fig. 5a). To obtain a single best estimate for the $45 \mathrm{~S}$ rDNA we combined estimates using Fisher's combined probability method (see Methods). The procedure identified 1,371 genes whose expression was significantly associated with rDNA dosage $(P<0.01$; false discovery rate $(\mathrm{FDR})=0.06$; Fig. $3 \mathrm{~b}), 198$ of which remain highly significant after a stringent Bonferroni correction $\left(P<6.4 \times 10^{-6} ; \quad \mathrm{FDR}=0.0002\right.$; Fig. 3b $)$. No chromosomal bias of significantly correlated genes was observed when tested against the distribution due to chance $\left(\chi^{2}\right.$ goodnessof-fit, degree of freedom $(\mathrm{DF})=22, P_{\text {more-stringent }}=0.48$ and $\left.P_{\text {less-stringent }}=0.77\right)$. Surprisingly, positive associations are significantly more abundant than negative associations and make up $>70 \%$ of correlated genes ( $\chi^{2}$ goodness-of-fit, $\mathrm{DF}=1$, $P_{\text {more-stringent }}=4.3 \times 10^{-18}$ and $\left.P_{\text {less-stringent }}=9.8 \times 10^{-5}\right)$. It is worth noting that we performed several analyses to ascertain the robustness of our inferences to confounders due to population structure, uncertainty in mRNA estimates and hidden sources of expression variation (see Methods). Most conservatively, we also identified rDNA-responsive genes independently within the CEU and YRI populations (Fig. $5 \mathrm{c}-\mathrm{e}$ ), and investigated patterns in an independently collected GE data set for the CEU population (see below). These analyses are in good agreement with one another (see section below), reinforce previous observations in Drosophila 8 and indicate that rDNA dosage is a major determinant of naturally occurring genome-wide GE variation in humans.
Expression responses to rDNA dosage are functionally coherent. Ribosome biogenesis involves a complex and concerted effort from $\sim 80$ ribosomal proteins and the hundreds of other ribosomal associated proteins, including transcription factors, enzymes, chaperones and nuclear export complexes ${ }^{26}$. The ribosome is assembled in the nucleolus, a dense nuclear compartment first recognized over two centuries $\mathrm{ago}^{27,28}$, and rDNA dosage might be particularly relevant for regulating genes functionally associated with this nuclear organelle. In agreement with our expectations, several genes whose expression is significantly and positively correlated with rDNA dosage are associated with the nucleolus. These include the following: RASL11A and DDX31, which are known regulators of rRNA expression; MAK16, which is involved in 60S ribosome biogenesis; NOP2, which is a putative methyltransferase, regulates the $25 \mathrm{~S}$ rRNA and influences $60 \mathrm{~S}$ assembly in yeast; NOP10, which is a small nucleolar ribonucleoprotein that may facilitate small nucleolar ribonucleoprotein assembly; and WDR74, which plays a crucial role in the processing and maturation of $18 \mathrm{~S} \mathrm{rRNA}^{29,30}$ (Fig. 5b). To further identify functionally coherent classes, we used the Gene Ontology (GO) annotation to categorize genes that are sensitive to $\mathrm{rDNA}$ dosage (see Methods). In agreement with our expectations, we observed that genes whose expression is positively associated with rDNA dosage are coherently clustered across several GO categories that are functionally related to the rDNA (Supplementary Table 2). 
a

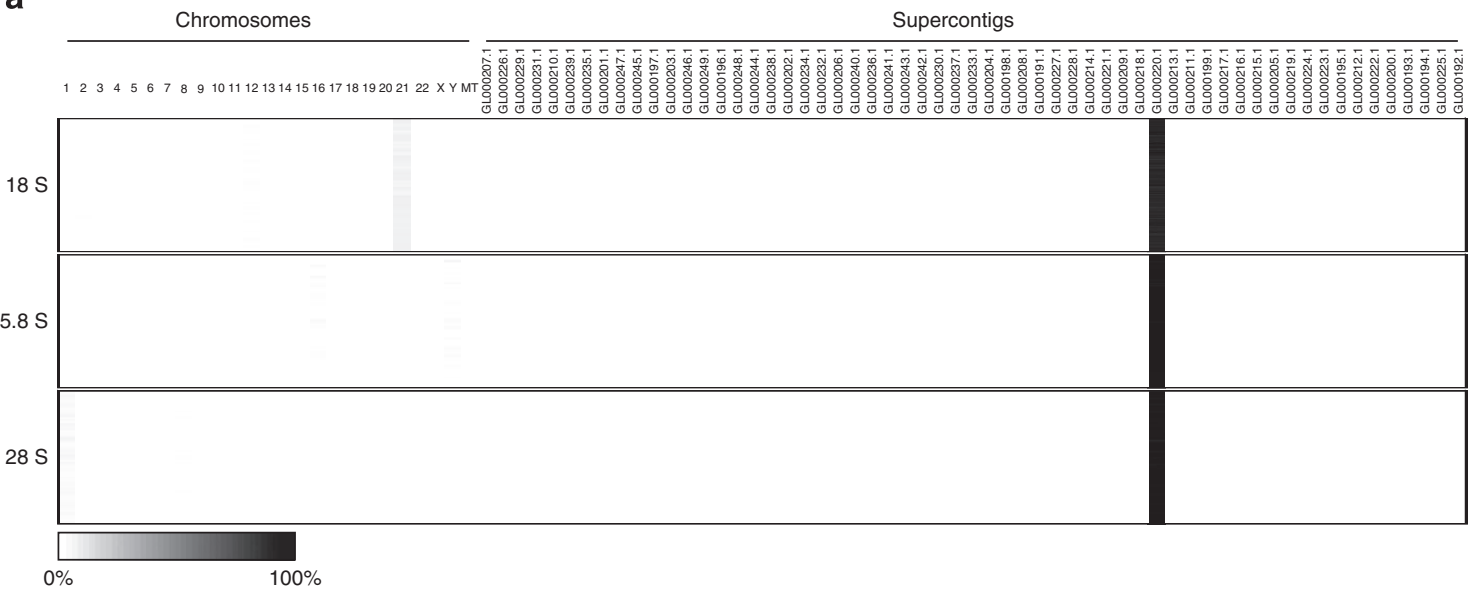

b

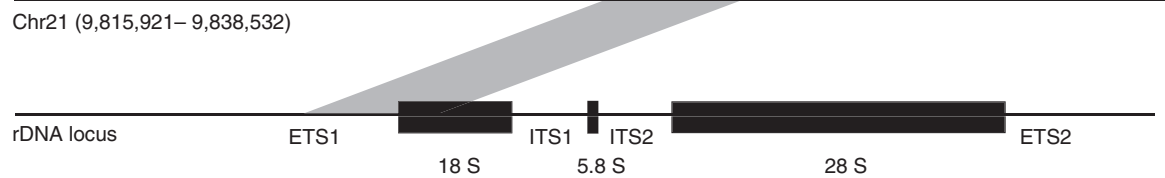

Figure 3 | rDNA reads map to the expected supercontigs and reveal a truncated rDNA array on chromosome 21. (a) Heat map depicting the percentage of reads mapping to each chromosome and supercontig of the human genome (hg19). For each individual $(N=168)$, the colour of the heat map corresponds to the percentage of rDNA reads mapped to each chromosome or supercontig, where white is zero and black is $100 \%$. The $y$ axis represents each individual and the $x$ axis represents chromosomes and supercontigs. The vast majority of rDNA reads map to the rDNA locus containing supercontig (GL000220.1). The fraction of reads correctly mapped to the expected supercontig was used as a correction factor. (b) Region of chromosome 21 $(9,815,921-9,838,532)$ to which $18 \mathrm{~S}$ reads map. The grey shaded area represents the region of homology between the segment of chromosome 21 and the rDNA locus.

Specifically, we observed significant enrichments in candidates localized to the nucleolus $\left(P=9.8 \times 10^{-4}\right)$, ribonucleoprotein complex $\left(P=2.2 \times 10^{-6}\right)$, ribosome $\left(P=2.4 \times 10^{-4}\right)$, mitochondrial ribosome $\left(P=3.0 \times 10^{-4}\right)$ and spliceosomal complex $\left(P=6.4 \times 10^{-4}\right.$; Fig. 6 and Supplementary Table 2$)$. The analyses also highlight significant enrichments in the processes of spliceosomal snRNP assembly $\left(P=8.5 \times 10^{-4}\right)$, RNA metabolism $\left(P=9.3 \times 10^{-4}\right), \quad$ ncRNa metabolism $\left(P=9.6 \times 10^{-4}\right)$ and response to virus $\left(P=6.9 \times 10^{-4}\right.$; Fig. 6a and Supplementary Table 2). Furthermore, the analyses point to a distinct set of genes negatively associated with rDNA dosage, with significant enrichment in candidates localized to the endoplasmatic reticulum $\left(P=1.8 \times 10^{-5}\right.$; Fig. $\left.6 a\right)$. Finally, consistent with the suggestion that rDNA dosage may alter genome-wide chromatin states $^{8}$, several genes with epigenetic roles in chromatin modification showed significant association with rDNA dosage, including CBX1 (HP1 $\beta), C T C F, C D Y L 2, M Y S T 1, R A S L 11 A$, CENPA, KTI12, INO8OC and KDM4B (Fig. 5b,c). Notably, HP1 $\beta$ is known to associate with the nucleolus and play a role in the transcription of ribosomal proteins ${ }^{31}$ and CTCF also localizes in the nucleolus and influences the chromatin state of rDNA arrays $^{32}$. Altogether, the data highlight coherent genomic responses to rDNA dosage, including a greater expression of ribosomal-associated proteins in response to higher rDNA dosage.

Additional analyses validate the functional coherence of loci that are sensitive to rDNA dosage. First, we observed that independent estimates are in good agreement between CEU and YRI populations, with the associations (genome-wide correlation coefficients) significantly correlated between populations (5.8S: $r=0.14, \quad P=7 \times 10^{-34}$ and 28S: $\left.\quad r=0.19, \quad P=5 \times 10^{-36}\right)$. Indeed, genes similarly associated with the $5.8 \mathrm{~S}$ in both CEU and YRI were enriched in targets that localize to the nucleolus
$\left(P=1.93 \times 10^{-4}\right)$, the ribosome $\left(P=4.36 \times 10^{-4}\right)$ and that belonging to the endoplasmatic reticulum membrane protein complexes $\left(P=7.87 \times 10^{-4}\right)$. Using this approach we identified, for instance, RASL11A, INO80C and MRPL44 as responsive to rDNA dosage (highlighted in Fig. 5b,c,e). These observations indicate that the major functional associations can be replicated independently within each population. Second, we performed association analysis on a subset of 27 overlapping CEU individuals for which RNA-seq data were generated from another study and processed through the same analysis pipeline ${ }^{33,34}$. We observed good agreement between expression values for biological replicates used between the studies (average Pearson correlation $(r)$ between expression values $=0.87$ ). Despite the much smaller size of the Cheung et al. ${ }^{33}$ data set, the association between rDNA dosage and GE were significantly correlated between studies (correlations between the strength of the association uncovered with expressed data from the Cheung et al. ${ }^{33}$ study and from the Montgomery et al. ${ }^{24}$ study: $18 \mathrm{~S}$ : $r=0.13, \quad P=5 \times 10^{-28} ; \quad 5.8 \mathrm{~S}: \quad r=0.23, \quad P=6 \times 10^{-73} ; \quad 28 \mathrm{~S}$ : $r=0.21, P=1 \times 10^{-58}$; mtDNA: $\left.r=0.47, P=4 \times 10^{-301}\right)$ and functional analysis similarly suggested an enrichment in candidates that localize to the mitochondria (mitochondrial part and mitochondrion; $P<8 . \times 10^{-4}$ for both categories). Lastly, patterns of functional enrichment were highly concordant between the PCA normalized and non-PCA normalized CEU expression data (Supplementary Tables 3 and 4). Altogether, our results with independently measured expressions levels from the same cells and with replication across populations lend support to the conclusion that the trans-regulatory consequence of rDNA dosage is pervasive and coherently manifested.

rDNA dosage is negatively associated with mtDNA abundance. Several studies indicate a close relationship between the rDNA 
a

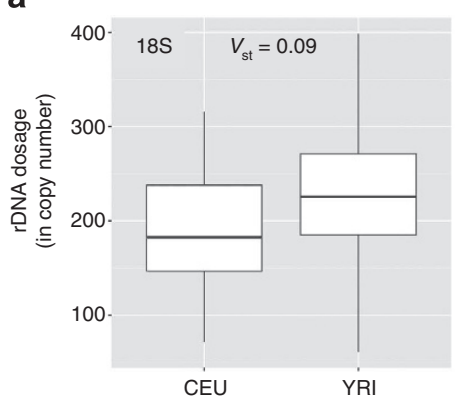

b

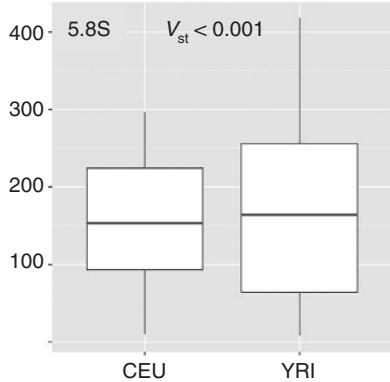

C

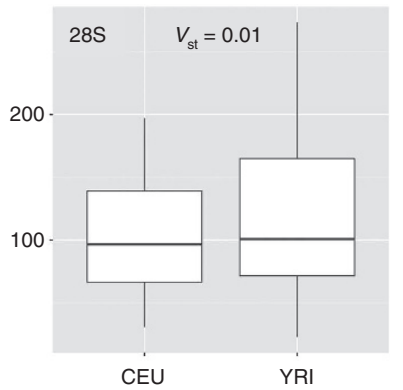

d

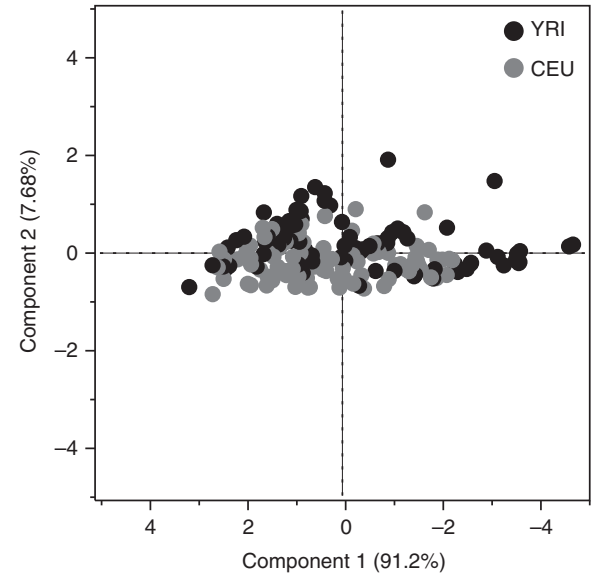

e

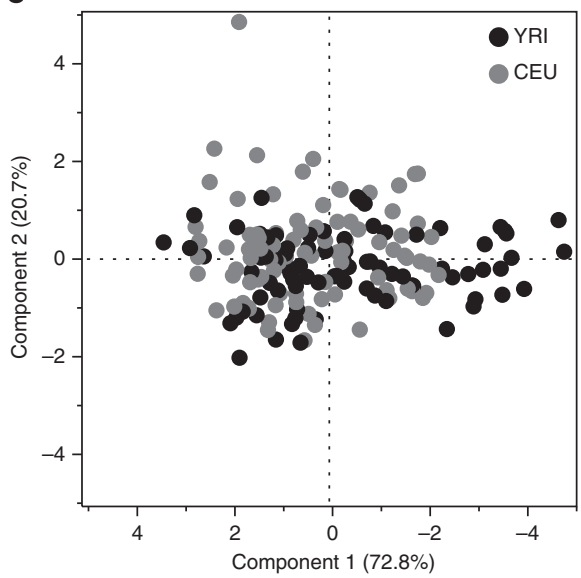

Figure 4 | rDNA dosage do not display population differentiation. $V_{S T}$ values for the (a) $18 \mathrm{~S}$, (b) $5.8 \mathrm{~S}$ and (c) $28 \mathrm{~S}$ rDNA components ( $N=168$ ). CN is depicted on the $y$ axis, while the CEU and YRI populations are shown on the $x$ axis. Box plots display the median, the 25th and 75th percentiles, and maximum and minimum. PCA analysis of (d) $45 \mathrm{~S}$ rDNA component (18S, 5.8S and 28S) CNs, (e) 45S and mtDNA abundance ( $N=168$ ). The $x$ and $y$ axes represent the first two principal components. Grey and black symbols represent the CEU and YRI individuals, respectively.

arrays, ribosomal biogenesis and the mitochondria ${ }^{10-13}$. For instance, recent evidence points to the import of $5 \mathrm{~S}$ rRNAs into the mitochondria through a highly regulated process $s^{35,36}$. Moreover, tight regulation of nuclear transcription ensures proper mitochondrial biogenesis ${ }^{11,13}$. As such, functional crosstalk between the rDNA dosage and the mitochondria might be manifested in natural populations. Indeed, the association between rDNA gene dosage and mitochondria is consistent with the overrepresentation of mitochondrial ribosome (Fig. 6a) genes in the set of candidates positively associated with rDNA dosage. Furthermore, the expression of 25 of the 50 nuclear genes encoding mitochondrial proteins analysed was significantly positively associated with rDNA dosage (at the $P$-value threshold of 0.01 ; see ' $M R P$ ' genes in Fig. 5). Altogether, the data indicate that rDNA gene dosage might influence mitochondrial-related processes. To address this issue, we quantified mtDNA abundance using an approach similar to that used for estimating rDNA dosage (Fig. 1, see Methods). Accordingly, we observed $\sim 4$-fold variation in mtDNA abundance among individuals, ranging from 226 to 1,074 mtDNA per diploid nuclear genome. Remarkably, mtDNA abundance was significantly negatively associated with dosage of all rDNA components $(n=168 ; \operatorname{mtDNA}$ versus 18S: Pearson's $r=-0.29, \quad P=1.3 \times 10^{-4}, \mathrm{mtDNA}$ versus $5.8 \mathrm{~S}$ : Pearson's $r=-0.34, \quad P=5.4 \times 10^{-6}$ and mtDNA versus 28S: $r=-0.36, P=1.2 \times 10^{-6}$; Fig. $\left.7 \mathrm{a}\right)$. Our results point towards interactions between $\mathrm{rDNA}$ and the mitochondria that is manifested across human genotypes. We find that rDNA dosage (i) influences the expression of mitochondrial-related genes (Figs 5b,d,e and 6a) and (ii) is negatively associated with mtDNA abundance (Fig. 7a). These results might reflect the synchronization of GE between the nuclear and mitochondrial genomes in a concerted effort to control proper levels of protein production for mitochondria assembly and intracellular homeostasis. Retrograde signalling is an essential mechanism in which organelles communicate with the nucleus to convey a response from fluctuating environmental or cellular conditions ${ }^{10}$. Indeed, we found that genes whose expression is negatively correlated with rDNA dosage were functionally enriched for carbonyl reductase (NADPH) activity (Fig. 6a). This observation is particularly noteworthy because NADPH oxidases are involved in the mitochondria-nucleus cross-talk ${ }^{10}$.

Mitochondrial DNA abundance and genome-wide expression. Finally, we evaluated the relationship between mtDNA abundance and nuclear GE, and identified 685 genes significantly correlated with mtDNA abundance $(P<0.01, \mathrm{FDR}=0.07), 14$ of which remained significant after a stringent Bonferroni-corrected threshold (Fig. 7b). Interestingly, over half the genes whose expression is associated with mtDNA abundance (359 genes) also showed significant association with rDNA dosage $(P<0.01$; Fig. 7c). The overlap was substantially greater than expected by chance $\left(\chi^{2}\right.$ goodness-of-fit, $\left.\mathrm{DF}=1, P=3.9 \times 10^{-107}\right)$. However, in contrast to that observed with rDNA dosage, negative associations between mtDNA abundance and nuclear GE were significantly more prevalent than positive associations ( $\chi^{2}$ goodness-of-fit, $\mathrm{DF}=1, P=1.8 \times 10^{-6}$ ). Indeed, all of the overlapping genes were inversely associated (that is, positively correlated with rDNA dosage and negatively correlated with mtDNA abundance, or negatively correlated with rDNA dosage and positively correlated with mtDNA abundance; Fig. 7d). 


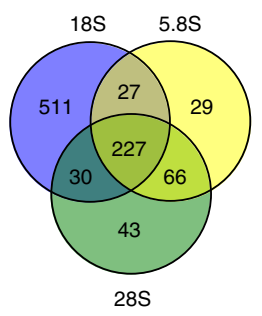

b

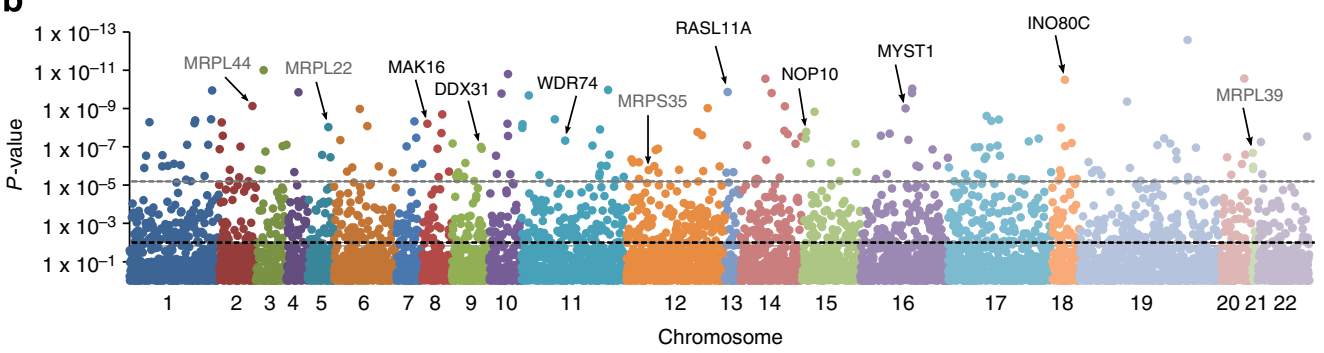

C

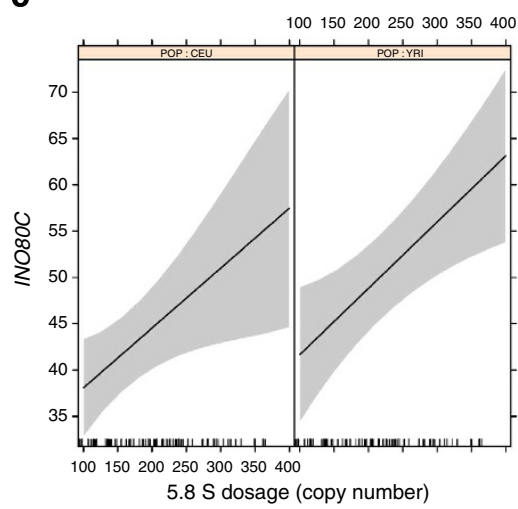

d

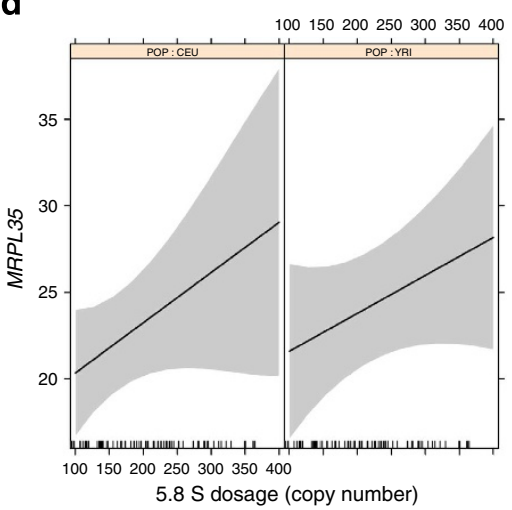

e

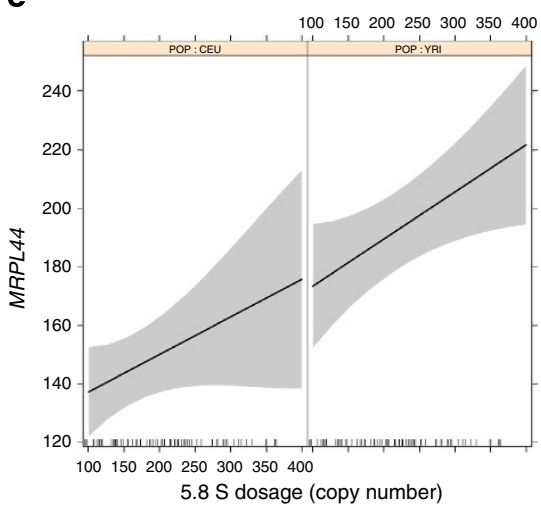

Figure 5 | rDNA dosage is a major trans-regulatory eQTL (Ribo-eQTL). (a) Independent associations between components of the 45S rDNA share significant overlap. Venn diagram of genes significantly correlated ( $P$-value $<0.01$ ) with rDNA dosage and GE. (b) Manhattan plot of genome-wide association results between $45 \mathrm{~S}$ rDNA CN and GE. Colours represent chromosomes and the Fisher's combined Pearson's correlation probability for the 18S, $5.8 \mathrm{~S}$ and $28 \mathrm{~S}$ rDNA components is depicted on the $y$ axis. The dotted black line represents the $P$-value significance thresholds of 0.01 , while the grey line represents the Bonferroni multiple test corrected $P$-value significance threshold of $6.4 \times 10^{-6}$. Examples of specific genes are labelled. (c-e) Examples of associations between $5.8 \mathrm{~S}$ dosage and GE in CEU and YRI populations. (c) INO80C is a chromatin modifying gene, while (d) MRPL35 and (e) MRPL44 are mitochondrial ribosomal genes. rDNA dosage and GE levels are represented on the $x$ and $y$ axes, respectively. The grey area represents the $95 \%$ intervals for the linear coefficients.

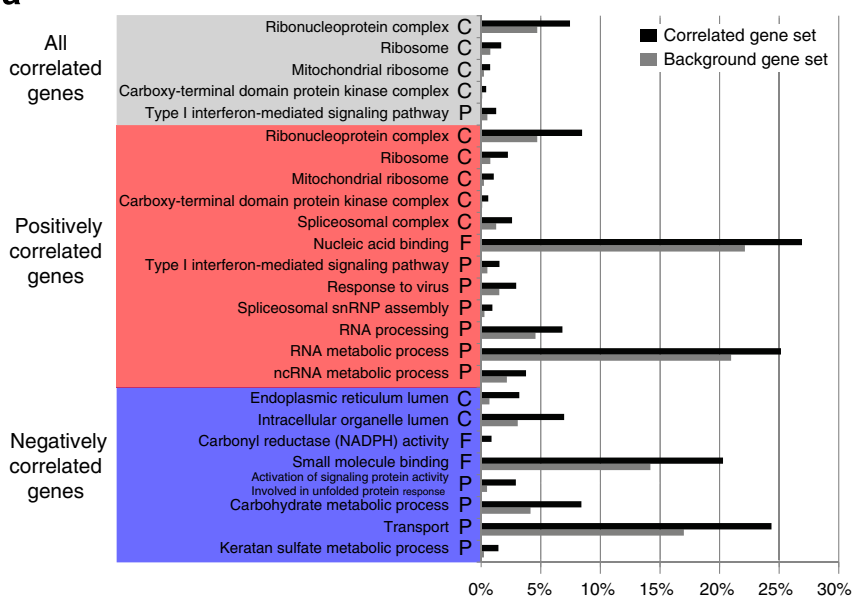

b

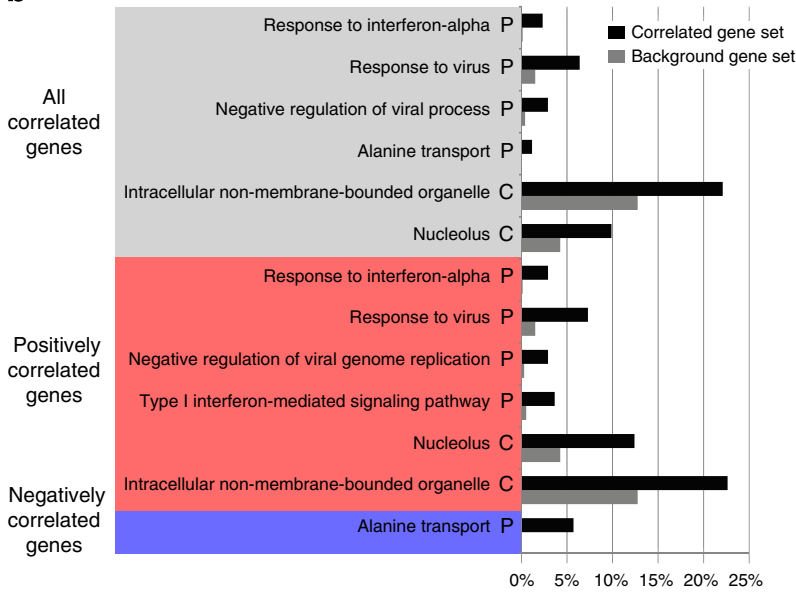

Figure 6 | rDNA dosage is associated with the expression of functionally coherent genes. GO functional enrichment analysis of correlated genes for significance thresholds of (a) $P<0.01$ and (b) $P<6.4 \times 10^{-6}(N=97)$. Redundant GO terms were reduced for clarity of display (see Supplementary Table 1 for complete GO term enrichment). Grey, red and blue shaded boxes represent GO term enrichment for all correlated, positively correlated and negatively correlated genes, respectively. Black and grey bars represent the percentages of correlated and background genes classified in each GO term, respectively. For all enriched GO terms $P$-value $<1 \times 10^{-3}$.

All eight of the mitochondrial ribosomal protein-encoding genes in the overlapping data set were positively correlated with rDNA dosage and negatively correlated with mtDNA abundance. None of the nuclear ribosomal protein-encoding genes was correlated with both the rDNA dosage and mtDNA abundance. Hence, the data indicate a balanced relationship between rDNA dosage and
mtDNA abundance that is manifested as an inverse association between these two elements in natural populations and divergent associations with genome-wide levels of expression. Interestingly, we also observed significant correlations between both rDNA dosage (positive) and mtDNA abundance (negative) with the mitochondrial transcription termination factor 3 (MTERF). 
a

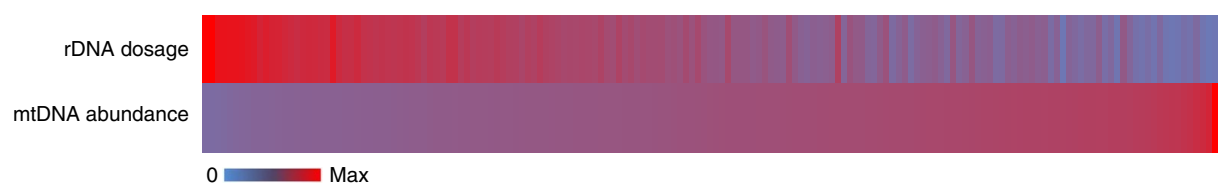

b

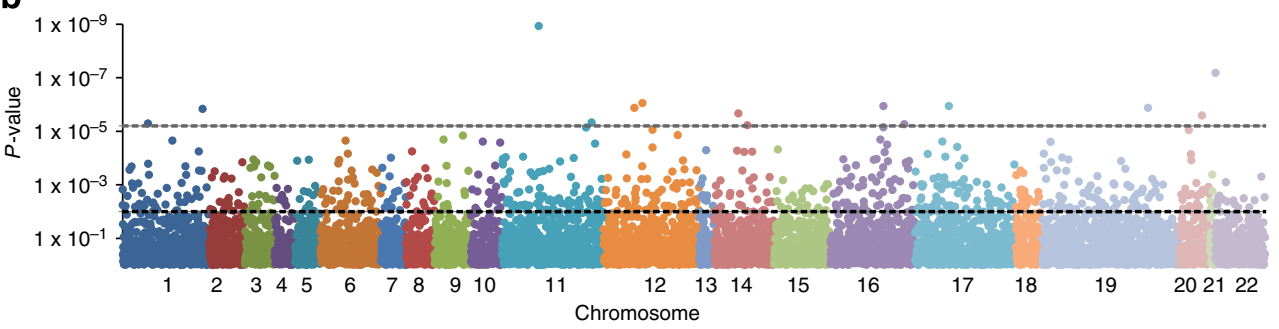

C d

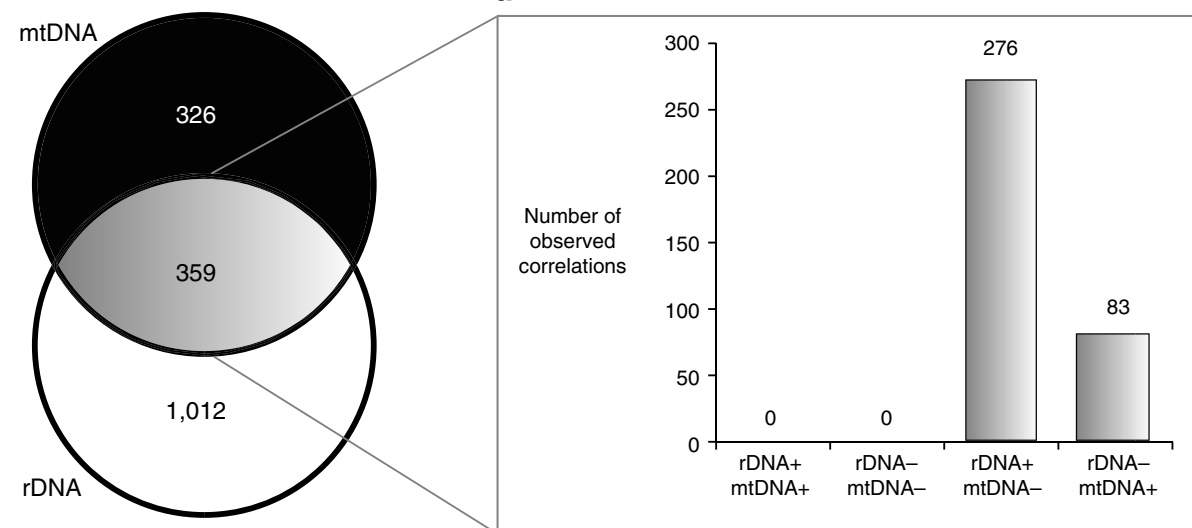

Figure 7 | rDNA dosage and mitochondrial DNA abundance are inversely correlated. (a) rDNA CN and mtDNA abundance are inversely correlated. rDNA dosage (average $\mathrm{CN}$ of the $18 \mathrm{~S}, 5.8 \mathrm{~S}$ and $28 \mathrm{~S}$ components) and mtDNA abundance are depicted for each of the 168 individuals. Blue and red correspond to zero and the measurement maximum, respectively. Individuals are sorted in ascending order of mtDNA abundance $(N=168)$. (b) Manhattan plot of genome-wide association between mtDNA CN and GE ( $N=97)$. The dotted black line represents the significance threshold of $P<0.01$, while the grey line represents the Bonferroni multiple test corrected $P$-value significance threshold of $6.4 \times 10^{-6}$. (c) Venn diagram showing significant overlap (grey) between genes whose expression is correlated with mtDNA (black) or rDNA (white). (d) All genes displaying association with both mtDNA and rDNA are inversely correlated. The $x$ axis represents the class of each overlapping correlated gene; ' + ' and ' - 'symbols depict positively and negatively correlated genes, respectively, and the $y$ axis provides the frequency of each occurrence.

This protein regulates mitochondrial transcription and mitochondrial ribosome biogenesis ${ }^{12}$. These results suggest tight regulatory relationship between $\mathrm{rDNA}$ dosage, GE of ribosomal proteins and mtDNA abundance, which emerge in the context of naturally occurring variability among individuals (Fig. 8).

\section{Discussion}

All aspects of cellular growth and development are dependent on ribosome biogenesis and protein synthesis. rRNA molecules make up the majority of eukaryotic ribosomes and, unlike mRNA transcripts, cannot be recycled through multiple rounds of amplification via translation. Hence, high $\mathrm{CN}$ rDNA arrays are expected to provide a sufficient source of cellular rRNAs to maintain a viable pool of rRNA molecules ${ }^{37}$. It is not surprising that sequence and structural rDNA polymorphisms can have drastic phenotypic consequences and association with disease ${ }^{7,38}$. Here we provide computational estimates of rDNA dosage and mtDNA abundance across hundreds of human genomes. Our analyses show abundant variation in rDNA dosage with regulatory properties (Ribo-eQTL) that functionally interact with genome-wide GE and mtDNA abundance.

In spite of the high level of sequence and functional conservation of rRNA molecules among eukaryotes, $\mathrm{CN}$ of the
rDNA locus is variable both within and between species ${ }^{38}$ Indeed, eukaryotic genomes display dramatic differences in rDNA dosage ranging from as few as $\sim 20$ to as many as 20,000 copies $^{39}$. Extensive epigenetic regulation of the rDNA loci further contributes to the complexity, with greater rDNA dosage not directly equating to higher expression levels of rRNA transcripts. Accordingly, an estimated $\sim 50 \%$ of rDNA loci are transcriptionally inactivated ${ }^{40}$ and further, total rRNA transcription can be compensated by upregulating individual rDNA loci rather than the activation of additional copies of the locus ${ }^{41}$. One established notion is that the functionally redundant rDNA copies in the genome provide more resources to transcribe rRNAs than what is typically required for ribosome biogenesis in actively growing cells ${ }^{38}$.

However, lower limits of rDNA dosage are evident. For example, in chicken strains, fewer than half of wild-type rDNA dosage caused embryonic lethality ${ }^{42}$, while in yeast, isolates with experimentally reduced rDNA dosage experience a gradual return to original levels over time ${ }^{43}$. On the other hand, upper limits placing boundaries of rDNA dosage might be less sharply defined. Rapid amplification of the locus has long been well documented in somatic tissues of fruitflies that inherit reduced rDNA $\operatorname{arrays}^{44-47}$, and an upwards of 10,000-fold amplification of the 


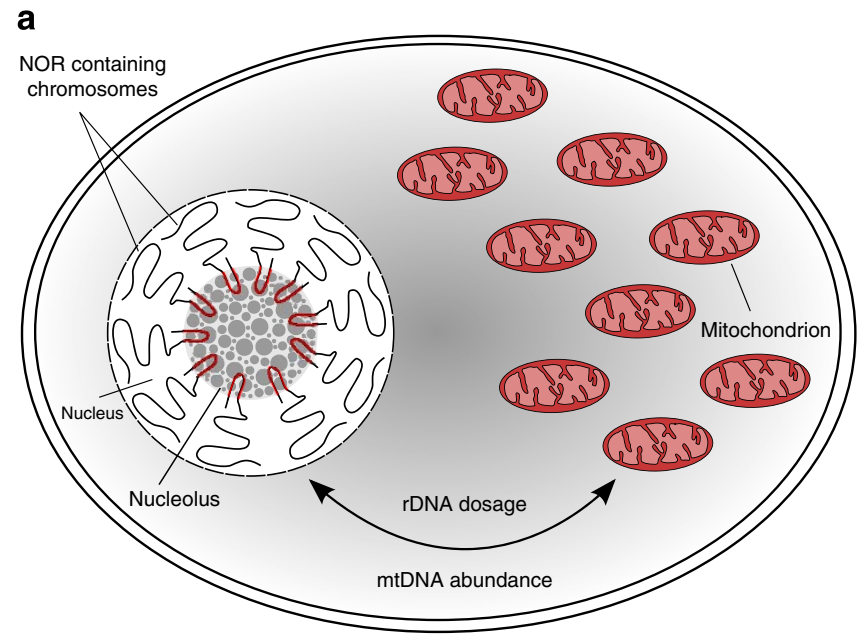

b

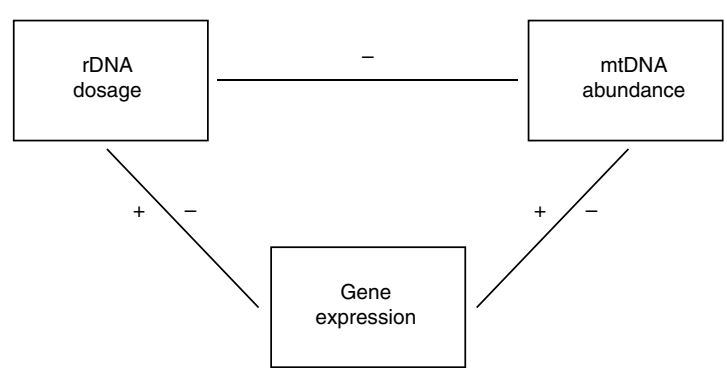

Figure 8 | Model summary with interactions between rDNA dosage and mtDNA abundance. (a) Within the eukaryotic nucleus, variable sized nucleolar organizing regions (NORs) localize to the nucleolus, associate with the expression of hundreds of genes and negatively correlate with mtDNA abundance. (b) Qualitative summary of the sign (negative or positive) of the associations herein uncovered. rDNA dosage and mtDNA abundance display inverse associations with GE (that is, genes with expression positively correlated with rDNA dosage and negatively correlated with mtDNA abundance or gene with expression negatively correlated with rDNA dosage and positively correlated with mtDNA abundance). These associations emerge at the population level as naturally occurring variation among individuals.

rDNA has been well described in amphibians oocytes ${ }^{48,49}$. These observations, along with others, indicate that somatic gene amplification and selection might contribute to shaping rDNA dosage $^{38}$ and that molecular models for the trans-regulatory effects of rDNA dosage need to be refined. In any circumstance, the associations between GE and dosage variation in the rDNA might point to molecular requirements and trade-offs that place boundaries on the range of rDNA dosage typically observed. We expect that, via the alteration of epigenetic states across the genome, these trade-offs might contribute to intragenomic adaptations with relevance to shaping optimal ranges for rDNA dosage.

Our results also show that polymorphic dosage variation in the rDNA locus interacts with the remaining of the genome beyond the production of rRNAs for ribosome biogenesis. Epigenetic models of chromatin dynamics ${ }^{50}$ that postulate a finite supply of dosage-sensitive chromatin regulators and DNA-binding proteins in the nucleus might explain the trans-regulatory modulation by Ribo-eQTL. These could involve, for instance, differential recruitment of chromatin-modifying proteins to rDNA arrays with variable $\mathrm{CN}$. Disproportional binding to rDNA locus might result in altered concentrations throughout the rest of the genome, thereby effecting genome-wide chromatin environments and transcription rates ${ }^{8,51-53}$. The observation that rDNA dosage is correlated with the expression of several proteins that modify the chromatin (Fig. 5b,c) is consistent with such a model. If these proteins are sequestered through preferentially binding to elements of the rDNA loci ${ }^{42,43}$, limited availability elsewhere in the genome might be expected to cause upregulation of GE in individuals with greater rDNA dosage. Such source-sink mechanisms might explain the strong biases for positive associations between GE and rDNA dosage.

Our study revealed substantial variation in rDNA dosage among individuals, ranging from as low as approximately ten to hundreds of copies that function as a major trans-regulatory modifier (Ribo-eQTL) of genome-wide expression and mitochondria abundance (Fig. 8). Limited availability of transcription factors or chromatin regulators required for rRNA transcription and for maintaining the epigenetic architecture of the rDNA loci might be one mechanism to account for genome-wide consequences of rDNA dosage. Our findings uncover a novel source of functional genomic variation in rDNA dosage that is hypervariable and revealed through the expression of functionally coherent sets of gene across the entire genome. They raise the question of how pervasive these consequences might be to other traits, including organismal fitness, cellular adaptation and disease phenotypes. Several examples of adaptive CNV are present in human populations ${ }^{54}$. In line with these patterns, variation in rDNA dosage might conceivably provide a molecular mechanism for cellular homeostasis, as well as a rapid and reversible source of variation for adaptation at the cellular and organismal levels.

\begin{abstract}
Methods
Human population genome data. Raw fastq whole-genome DNA Illumina sequence reads for individuals with estimated coverage $>5 \times$ were obtained from the 1,000 Genomes Project FTP site (http://1000genomes.org) ${ }^{14}$. Approximate sequence read coverage was estimated pre-mapping by multiplying read length by read count. We focused on individuals from the CEU and YRI populations, as they represented populations with substantial, high-coverage samples. We quality trimmed reads from each sample using Trim Galore (http://www.bioinformatics. babraham.ac.uk/projects/trim_galore/). Residual adapter sequences were removed from reads, and reads were trimmed such that they contained a minimum quality score of 20 at each nucleotide position. Trimmed reads shorter than 50 nucleotides were discarded.
\end{abstract}

Reference sequences. The consensus $43 \mathrm{~Kb}$ human $\mathrm{rDNA}$ reference sequence was obtained from GenBank (accession: U13369) ${ }^{17}$. We modified this sequence to include $\mathrm{a} \sim 17.65$-kb region of the rDNA locus extending $2 \mathrm{~kb}$ upstream of the core promoter region to $2 \mathrm{~kb}$ downstream of the ETS2 region (Fig. 1b). The entire human mitochondrion genome was downloaded from the NCBI Reference Sequence Database (accession: NC_012920.1)

The complete human exon reference was downloaded from The European Bioinformatics Institute (ftp://ftp.ebi.ac.uk/pub/databases/astd/current_release/ human/9606_exons.fa.gz). To retain putative single-copy exons, we performed several layers of filtering. First, exons with significant BLAST hits ( $E$-value $\left.<1 \times 10^{-6}\right)^{56}$ to any other exons were removed to reduce potentially ambiguous regions. Second, only the largest exon of each gene was retained to avoid mapping to alternatively transcribed exons with overlapping regions. Third, only exons $\geq 300$ bp were retained to reduce mapping biases to short target regions. Our final single-copy exon reference data set consisted of 12,453 sequences.

The complete set of human introns was downloaded from the UCSC Genome Table Browser. We performed similar, but additional, filtering as the exons to obtain a reliable set of single copy introns: (i) introns with significant BLAST hits ( $E$-value $<1 \times 10^{-6}$ ) to any other introns were removed, (ii) only the largest intron of each gene was retained, (iii) only the first intron located within $10 \mathrm{~kb}$ of any other intron was retained, (iv) only introns $\geq 300$ bp were retained, (iv) only introns $\leq 10 \mathrm{~kb}$ were retained. This filtering resulted in an intron data set consisting of 1,131 introns.

Read mapping. Filtered read sets for each sample were mapped against the reference rDNA locus, mitochondrion genome and sets of single-copy exons and introns using Bowtie2 v2.0.5 (ref. 57). Reads were mapped in unpaired fashion using the 'sensitive' preset (full command line parameters: --end-to-end -D 15 -R 2 -L 22 -i S,1,1.15). Mapping output was converted to sorted bam format using the samtools (v0.1.18) 'view' and 'sort' functions, respectively ${ }^{58}$. 
Background read depth estimation. BRD was calculated for each sample and was used as a normalizing factor to estimate rDNA component dosage and mtDNA abundance (Fig. 1c-e). Per-base BRD was independently calculated from the exon and intron sorted bam mapping output files using the samtools 'depth' function ${ }^{58}$. To correct for artificial mapping reduction in the beginning and end of reference sequences, per-base depth values from the first and last $108 \mathrm{bp}$ (longest read length in data set) were excluded. For each sample, we excluded read depth values in the upper 5\% of the distribution. Exon and intron BRD estimates were nearly identical $\left(r^{2}=0.99\right.$; Supplementary Fig. 1$)$. Hence, we used the average of the two values to represent consensus BRD.

rDNA dosage and mtDNA abundance estimation. Consensus BRD was used as a normalizing factor to represent the read depth of an autosomal locus with a diploid $\mathrm{CN}$ of two. Average read depth of each rDNA component was also calculated using the samtools 'depth' function (Fig. 1c). For each sample, rDNA component dosage was calculated as:

$$
\text { rDNA Component Dosage }=\frac{\text { rDNA Component ARD }}{\text { BRD }}
$$

where ARD is the average read depth across all sites in the rDNA component and $\mathrm{BRD}$ is the background read depth. Stability in CN variable loci has been demonstrated in DNA extracted from lymphoblastoid cell lines versus whole blood $^{59-61}$. We used the same approach to estimate mtDNA abundance as the average mtDNA read depth normalized by the BRD. Homology between nuclear and mitochondrial ribosomal genes did not bias read depth for either the rDNA dosage or mtDNA abundance estimates, as none of the reads that mapped to the rDNA locus also mapped to the mitochondrion genome. The length of the targeted sequence (rDNA component and mtDNA genome) does not influence the estimates of read depth.

Robustness of rDNA read mapping. CN and dosage estimations are sensitive to read mapping. Sequence similarity between rDNA components could potentially confound read depth coverage and thus rDNA dosage quantification. We assessed sequence similarity between rDNA components by BLASTing each rDNA component against all other rDNA components $\left(E \text {-value }<1 \times 10^{-6}\right)^{56}$. We also assessed sequence homology by aligning all pairwise combinations of rDNA components using CLUSTALW (default settings) ${ }^{62}$. Furthermore, we tested whether rDNA reads mapped to multiple rDNA components, as such a phenomenon could confound rDNA sequence read depth values. Reads that mapped to each component were independently extracted using the 'samtools view' function. Reads for each rDNA component were then mapped against all other rDNA components using the 'sensitive' option in Bowtie2 v2.0.5 (ref. 57).

Correction factor for estimating dosage of rDNA components. We tested whether rDNA reads aberrantly mapped to alternative regions of the genome (potential rDNA pseudogenes). This effect could inflate rDNA read depth values and rDNA dosage estimates. To test this issue, extracted reads from each rDNA component were independently mapped against the reference GRCh37 (hg19) human genome using Bowtie2 v2.0.5 (ref. 57). The number of reads mapping to each chromosome and supercontig were calculated from the mapping output bam file using the samtools 'idxstats' function. For each sample, and each component, we applied a correction factor to our original estimates of rDNA dosage by multiplying each rDNA component dosage by the percentage of reads mapping to expected chromosomes or supercontigs (chromosomes 13, 14, 15, 21, 22 and supercontig GL000220.1).

Validation of $\mathbf{C N}$ estimates. To validate our method of CN quantification, we measured CN of 6 genes previously characterized in 80 overlapping individuals ${ }^{16}$. For the 80 samples, entire read sets were mapped against the CCL3L1, TBC1D3F, MBD3L3, USP17L6P, FAM157B and PRR20A genes using Bowtie2 v2.0.5 (ref. 57). As $B R D$ was nearly identical between exons and introns, we used the entire gene, from start codon to stop codon (including introns), for mapping. CN for each gene was calculated as described for the rDNA dosage quantification. For each gene, our results were plotted against results from Sudmant et al. ${ }^{16}$ Linear regression analysis was used to assess the correlation of $\mathrm{CN}$ quantification between studies.

Population structure and differentiation of rDNA dosage. CEU and YRI display relatively small levels of population differentiation relative to other human population pairs ${ }^{63}$. It is also beneficial to our purposes that there is no evidence for population stratification within either CEU or YRI populations $s^{63,64}$. To further examine population differentiation specifically in rDNA dosage between CEU and YRI populations, we calculated $V_{\mathrm{ST}}$ values for each rDNA component ${ }^{60} . V_{\mathrm{ST}}$, analogous to $F_{\mathrm{ST}}$, compares the variance in CNV within and between populations. $V_{\mathrm{ST}}$ was calculated as $\left(V_{\mathrm{T}}-V_{\mathrm{S}}\right) / V_{\mathrm{T}}$, where $V_{\mathrm{T}}$ is the variance in $\log _{2}$ ratios among all unrelated individuals and $V_{S}$ is the population size-weighted average variance within each population (Fig. 4a). In addition, we used PCA analysis to assess multivariate population differentiation in these loci. PCA was independently performed using the dosage estimates for the set of $45 \mathrm{~S}$ rDNA components
(18S, $5.8 \mathrm{~S}$ and $28 \mathrm{~S}$ ), and the set of $45 \mathrm{~S}$ components and the estimates of mtDNA abundance (Fig. 4d,e).

Genome-wide association of rDNA dosage on GE. Normalized mapped read results were obtained from the ReCount database (http://bowtie-bio.sourceforge.net/recount//34 from two previous RNA-seq studies of lymphoblastoid cell lines 24,25 for 97 individuals with matched rDNA CN measurements (29 female CEU, 25 male CEU, 31 female YRI and 12 male YRI). For each sample, GE was measured as:

$$
\mathrm{GE}=\frac{\text { Genes uniquely mapped to transcript }}{\text { Total number of uniquely mapped reads in experiment }} * 1,000,000
$$

We constrained our analysis to genes that were expressed in at least 50 samples, resulting in 7,841 genes with high-quality estimates of expression.

Pearson's product-moment correlation coefficient $(r)$ was used to measure the association between rDNA CN and GE. Correlations were calculated for each rDNA component independently (18S, $5.8 \mathrm{~S}$ and $28 \mathrm{~S})$. We combined the probabilities of the 18S, $5.8 \mathrm{~S}$ and $28 \mathrm{~S}$ rRNA genes using Fisher's combined probability method ${ }^{65}$. The $18 \mathrm{~S}, 5.8 \mathrm{~S}$ and $28 \mathrm{~S}$ rRNA genes are encoded within the same locus, their dosage levels were highly correlated (Figs 1 and 2) and they share extensive overlap between significantly correlated genes (Fig. 5a,b). The relationship between mtDNA abundance and GE was estimated in the same manner. Finally, mixed effect linear models were used in the eQTL analyses.

To test whether significantly correlated genes were represented disproportionately in particular chromosomes, we compared the proportion of observed and expected genes present on each chromosome assuming no chromosomal bias using a $\chi^{2}$ goodness-of-fit test. To test whether significantly positively and negatively correlated genes were unevenly represented, we compared the observed frequencies to the expected frequencies assuming an equal distribution of correlation types. This hypothesis was tested using a $\chi^{2}$ goodness-offit test. Finally, we tested whether the number of overlapping rDNA and mtDNA correlated genes were more abundant than expected by chance using a $\chi^{2}$ goodness-of-fit test. The number of observed and expected overlapping genes and background genes were compared. The expected number of overlapping genes were calculated as total number of analysed genes $\times$ relative frequency of mtDNA significantly correlated genes $\times$ relative frequency of rDNA significantly correlated genes. Expected background genes were calculated as total number of analysed genes - expected number of overlapping genes.

PCA analysis of expression estimates. To account for factors that might influence global patterns of GE, we performed PCA analysis on the sample correlation matrix independently for each population. The first three principal components accounted for $>95 \%$ of the variance in both CEU and YRI populations. We have computed the residuals from the regression between the first three principal components and each of the original estimates of expression values. These residuals were used as input for the eQTL analysis. The stability of the eQTL results between the original normalized expression estimates and the PC residuals were investigated in two ways. First, we compared the estimates of association in both analyses (that is, the stability of the Pearson correlations). Second, we carefully inspected the stability of functionally coherent gene sets. Overall, the analysis showed strong concordance between the associations estimated directly with normalized expression values or estimated on the residuals after removal of the first three principal components (18S: $r=0.72,5.8 \mathrm{~S}: r=0.80$ and 28S: $r=0.80$; for all comparisons, $\left.P<1 \times 10^{-300}\right)$. Principal components were estimated and removed separately for each population. Population effects were estimated with linear models.

GO enrichment of gene sets. GO enrichment analysis was performed on subsets of genes using the GOrilla Gene Ontology enRIchment anaLysis and visuaLizAtion tool $^{66}$. For this analysis, GO enrichment was performed on the target set of genes compared with the background set of genes (7,841 analysed genes - target set of genes). Enrichment was independently assessed against the Biological Processes, Molecular Function and Cellular Component ontologies implementing a $P$-value threshold 0.001 .

\section{References}

1. Rabl, J., Leibundgut, M., Ataide, S. F., Haag, A. \& Ban, N. Crystal structure of the eukaryotic $40 \mathrm{~S}$ ribosomal subunit in complex with initiation factor 1. Science 331, 730-736 (2011).

2. Henderson, A. S., Warburton, D. \& Atwood, K. C. Letter: Ribosomal DNA connectives between human acrocentric chromosomes. Nature 245, 95-97 (1973).

3. Sorensen, P. D. \& Frederiksen, S. Characterization of human 5S rRNA genes. Nucleic Acids Res. 19, 4147-4151 (1991).

4. Fatica, A. \& Tollervey, D. Making ribosomes. Curr. Opin. Cell Biol. 14, 313-318 (2002). 
5. Lewis, J. D. \& Tollervey, D. Like attracts like: getting RNA processing together in the nucleus. Science 288, 1385-1389 (2000).

6. Warner, J. R. The economics of ribosome biosynthesis in yeast. Trends Biochem. Sci. 24, 437-440 (1999).

7. Narla, A. \& Ebert, B. L. Ribosomopathies: human disorders of ribosome dysfunction. Blood 115, 3196-3205 (2010).

8. Paredes, S., Branco, A. T., Hartl, D. L., Maggert, K. A. \& Lemos, B. Ribosomal DNA deletions modulate genome-wide gene expression: "rDNA-sensitive" genes and natural variation. PLoS Genet. 7, e1001376 (2011).

9. Satoh, M. \& Kuroiwa, T. Organization of multiple nucleoids and DNA molecules in mitochondria of a human cell. Exp. Cell Res. 196, 137-140 (1991).

10. Woodson, J. D. \& Chory, J. Coordination of gene expression between organellar and nuclear genomes. Nat. Rev. Genet. 9, 383-395 (2008).

11. Surovtseva, Y. V. et al. Mitochondrial ribosomal protein L12 selectively associates with human mitochondrial RNA polymerase to activate transcription. Proc. Natl Acad. Sci. USA 108, 17921-17926 (2011).

12. Wredenberg, A. et al. MTERF3 regulates mitochondrial ribosome biogenesis in invertebrates and mammals. PLoS Genet. 9, e1003178 (2013).

13. Zunino, R., Braschi, E., Xu, L. \& McBride, H. M. Translocation of SenP5 from the nucleoli to the mitochondria modulates DRP1-dependent fission during mitosis. J. Biol. Chem. 284, 17783-17795 (2009).

14. 1000 Genomes Project Consortium et al. An integrated map of genetic variation from 1,092 human genomes. Nature 491, 56-65 (2012).

15. Alkan, C. et al. Personalized copy number and segmental duplication maps using next-generation sequencing. Nat. Genet. 41, 1061-1067 (2009).

16. Sudmant, P. H. et al. Diversity of human copy number variation and multicopy genes. Science 330, 641-646 (2010).

17. Zentner, G. E., Saiakhova, A., Manaenkov, P., Adams, M. D. \& Scacheri, P. C. Integrative genomic analysis of human ribosomal DNA. Nucleic Acids Res. 39, 4949-4960 (2011).

18. Caburet, S. et al. Human ribosomal RNA gene arrays display a broad range of palindromic structures. Genome Res. 15, 1079-1085 (2005).

19. Zafiropoulos, A., Tsentelierou, E., Linardakis, M., Kafatos, A. \& Spandidos, D. A. Preferential loss of $5 \mathrm{~S}$ and $28 \mathrm{~S}$ rDNA genes in human adipose tissue during ageing. Int. J. Biochem. Cell Biol. 37, 409-415 (2005).

20. Eagle, S. H. \& Crease, T. J. Copy number variation of ribosomal DNA and Pokey transposons in natural populations of Daphnia. Mob. DNA 3, 4 (2012).

21. Stults, D. M., Killen, M. W., Pierce, H. H. \& Pierce, A. J. Genomic architecture and inheritance of human ribosomal RNA gene clusters. Genome Res. 18, 13-18 (2008).

22. Schmickel, R. D. Quantitation of human ribosomal DNA: hybridization of human DNA with ribosomal RNA for quantitation and fractionation. Pediatr. Res. 7, 5-12 (1973).

23. Paredes, S. \& Maggert, K. A. Ribosomal DNA contributes to global chromatin regulation. Proc. Natl Acad. Sci. USA 106, 17829-17834 (2009).

24. Montgomery, S. B. et al. Transcriptome genetics using second generation sequencing in a Caucasian population. Nature 464, 773-777 (2010).

25. Pickrell, J. K. et al. Understanding mechanisms underlying human gene expression variation with RNA sequencing. Nature 464, 768-772 (2010).

26. Staley, J. P. \& Woolford, Jr J. L. Assembly of ribosomes and spliceosomes: complex ribonucleoprotein machines. Curr. Opin. Cell Biol. 21, 109-118 (2009).

27. Montomgery, T. H. Comparative cytological studies, with especial regard to the morphology of the nucleolus. J. Morphol. 15, 265-582 (1898).

28. Pederson, T. The plurifunctional nucleolus. Nucleic Acids Res. 26, 3871-3876 (1998).

29. Andersen, J. S. et al. Nucleolar proteome dynamics. Nature 433, 77-83 (2005).

30. Boisvert, F. M., van Koningsbruggen, S., Navascues, J. \& Lamond, A. I. The multifunctional nucleolus. Nat. Rev. Mol. Cell Biol. 8, 574-585 (2007).

31. Horakova, A. H. et al. SUV39h-independent association of HP1 beta with fibrillarin-positive nucleolar regions. Chromosoma 119, 227-241 (2010).

32. van de Nobelen, S. et al. CTCF regulates the local epigenetic state of ribosomal DNA repeats. Epigenetics Chromatin 3, 19 (2010).

33. Cheung, V. G. et al. Polymorphic cis- and trans-regulation of human gene expression. PLoS Biol. 8 pii e1000480 (2010).

34. Frazee, A. C., Langmead, B. \& Leek, J. T. ReCount: a multi-experiment resource of analysis-ready RNA-seq gene count datasets. BMC Bioinformatics 12, 449 (2011).

35. Smirnov, A., Entelis, N., Martin, R. P. \& Tarassov, I. Biological significance of $5 \mathrm{~S}$ rRNA import into human mitochondria: role of ribosomal protein MRP-L18. Genes Dev. 25, 1289-1305 (2011).

36. Smirnov, A. et al. Mitochondrial enzyme rhodanese is essential for $5 \mathrm{~S}$ ribosomal RNA import into human mitochondria. J. Biol. Chem. 285, 30792-30803 (2010).

37. Prokopowich, C. D., Gregory, T. R. \& Crease, T. J. The correlation between rDNA copy number and genome size in eukaryotes. Genome 46, 48-50 (2003).

38. Weider, L. J. et al. The functional significance of ribosomal (r)DNA variation: impacts on the evolutionary ecology of organisms. Annu. Rev. Ecol. Evol. Syst. 36, 219-242 (2005)
39. Long, E. O. \& Dawid, I. B. Repeated genes in eukaryotes. Ann. Rev. Biochem. 49, 727-764 (1980).

40. Schlesinger, S., Selig, S., Bergman, Y. \& Cedar, H. Allelic inactivation of rDNA loci. Genes Dev. 23, 2437-2447 (2009).

41. Banditt, M., Koller, T. \& Sogo, J. M. Transcriptional activity and chromatin structure of enhancer-deleted rRNA genes in Saccharomyces cerevisiae. Mol. Cell Biol. 19, 4953-4960 (1999).

42. Delany, M. E., Muscarella, D. E. \& Bloom, S. E. Effects of rRNA gene copy number and nucleolar variation on early development: inhibition of gastrulation in rDNA-deficient chick embryos. J. Hered. 85, 211-217 (1994).

43. Kobayashi, T., Heck, D. J., Nomura, M. \& Horiuchi, T. Expansion and contraction of ribosomal DNA repeats in Saccharomyces cerevisiae: requirement of replication fork blocking (Fob1) protein and the role of RNA polymerase I. Genes Dev. 12, 3821-3830 (1998).

44. Bianciardi, A., Boschi, M., Swanson, E. E., Belloni, M. \& Robbins, L. G. Ribosomal DNA organization before and after magnification in Drosophila melanogaster. Genetics 191, 703-723 (2012).

45. Spear, B. B. \& Gall, J. G. Independent control of ribosomal gene replication in polytene chromosomes of Drosophila melanogaster. Proc. Natl Acad. Sci. USA 70, 1359-1363 (1973).

46. Tartof, K. D. Increasing the multiplicity of ribosomal RNA genes in Drosophila melanogaster. Science 171, 294-297 (1971).

47. Tartof, K. D. Regulation of ribosomal RNA gene multiplicity in Drosophila melanogaster. Genetics 73, 57-71 (1973).

48. Brown, D. D. \& Dawid, I. B. Specific gene amplification in oocytes. Oocyte nuclei contain extrachromosomal replicas of the genes for ribosomal RNA. Science 160, 272-280 (1968).

49. Gall, J. G. Differential synthesis of the genes for ribosomal RNA during amphibian oogenesis. Proc. Natl Acad. Sci. USA 60, 553-560 (1968).

50. Santoro, R., Li, J. \& Grummt, I. The nucleolar remodeling complex NoRC mediates heterochromatin formation and silencing of ribosomal gene transcription. Nat. Genet. 32, 393-396 (2002).

51. Case, L. K. et al. The Y chromosome as a regulatory element shaping immune cell transcriptomes and susceptibility to autoimmune disease. Genome Res. 23, 1474-1485 (2013).

52. Lemos, B., Araripe, L. O. \& Hartl, D. L. Polymorphic Y chromosomes harbor cryptic variation with manifold functional consequences. Science 319, 91-93 (2008).

53. Lemos, B., Branco, A. T. \& Hartl, D. L. Epigenetic effects of polymorphic Y chromosomes modulate chromatin components, immune response, and sexual conflict. Proc. Natl Acad. Sci. USA 107, 15826-15831 (2010).

54. Iskow, R. C., Gokcumen, O. \& Lee, C. Exploring the role of copy number variants in human adaptation. Trends Genet. 28, 245-257 (2012).

55. Andrews, R. M. et al. Reanalysis and revision of the Cambridge reference sequence for human mitochondrial DNA. Nat. Genet. 23, 147 (1999).

56. Altschul, S. F. et al. Gapped BLAST and PSI-BLAST: a new generation of protein database search programs. Nucleic Acids Res. 25, 3389-3402 (1997).

57. Langmead, B. \& Salzberg, S. L. Fast gapped-read alignment with Bowtie 2. Nat. Methods 9, 357-359 (2012).

58. Li, H. et al. The Sequence Alignment/Map format and SAMtools. Bioinformatics 25, 2078-2079 (2009).

59. Nickles, D. et al. In depth comparison of an individual's DNA and its lymphoblastoid cell line using whole genome sequencing. BMC Genomics 13, 477 (2012).

60. Redon, R. et al. Global variation in copy number in the human genome. Nature 444, 444-454 (2006).

61. Londin, E. R. et al. Whole-exome sequencing of DNA from peripheral blood mononuclear cells (PBMC) and EBV-transformed lymphocytes from the same donor. BMC Genomics 12, 464 (2011).

62. Thompson, J. D., Higgins, D. G. \& Gibson, T. J. CLUSTAL W: improving the sensitivity of progressive multiple sequence alignment through sequence weighting, position-specific gap penalties and weight matrix choice. Nucleic Acids Res. 22, 4673-4680 (1994).

63. Stranger, B. E. et al. Patterns of cis regulatory variation in diverse human populations. PLoS Genet. 8, e1002639 (2012).

64. International HapMap Consortium et al. A second generation human haplotype map of over 3.1 million SNPs. Nature 449, 851-861 (2007).

65. Sokal, R. R. Biometry: The Principles and Practice of Statistics in Biological Research (W. H. Freeman and Co., 2011).

66. Eden, E., Navon, R., Steinfeld, I., Lipson, D. \& Yakhini, Z. GOrilla: a tool for discovery and visualization of enriched GO terms in ranked gene lists. BMC Bioinformatics 10, 48 (2009).

\section{Acknowledgements}

We thank David C. Rinker and the SEQanswers community for helpful discussion, and Gabriel Zentner, Peter Sudmant, Evan Eichler, Tomas Marques-Bonet Javier Prado and 
Tuuli Lappalainen for access to data. We thank members of the Lemos lab and the anonymous reviewers for constructive comments. The computations in this paper were run on the Odyssey cluster supported by the FAS Science Division Research Computing Group at Harvard University. We thank support from training grant T32-HL007118. B.L. thanks the support of an Ellison Medical Foundation New Scholars in Aging Award and a Smith Family Award for Biomedical Research.

\section{Author contributions}

J.G.G. and B.L. designed the study. J.G.G., A.B., S.Y. and B.L. performed data analysis, and J.G.G. and B.L. wrote the manuscript.

\section{Additional information}

Supplementary Information accompanies this paper at http://www.nature.com/ naturecommunications

Competing financial interests: The authors declare no competing financial interests.

Reprints and permission information is available online at http://npg.nature.com/ reprintsandpermissions/

How to cite this article: Gibbons, J. G. et al. Ribosomal DNA copy number is coupled with gene expression variation and mitochondrial abundance in humans. Nat. Commun. 5:4850 doi: 10.1038/ncomms5850 (2014). 